\title{
An Efficient Scheme for MIMO Broadcast Channels Based on Iterative User Selection
}

\author{
Yingbo Li, Wei Jiang, Jianjun Zhang, Zhongjian Liu, and Ye Jin
}

\begin{abstract}
Dirty paper coding (DPC) is shown to achieve the capacity of multiple-input multiple-output (MIMO) Gaussian broadcast channels (BCs). In circuit data scenarios, users demand to get service at fixed desire rate. Finding the optimal covariance matrices and order of users for maximizing user capacity requires high complexity computation. To deal with this problem, many researchers use fixed order of users and get subpar performance. In this paper we present an iterative user selection scheme that aims to maximize user capacity. The main feature of the proposed scheme is that it do not impose a fixed order on the users. Users to be served are chosen iteratively considering influence from the chosen ones. Simulation results show that the proposed scheme achieves better performance than similar simple schemes. In addition, a compromise between performance and complexity can be made by a candidate user set concept. Cardinality of the candidate user set is fixed and users in the set are dynamically adjusted.
\end{abstract}

Index Terms-DPC, Iterative User Selection, MIMO gaussian broadcast channel, user capacity.

\section{INTRODUCTION}

Multiple-input multiple-output (MIMO) techniques, by implementing multiple antennas at both the transmitter and receiver, have receive a lot of attention, since the heuristic research works by Telatar [1] and G. J. Foschini [2]. MIMO technology is considered as a promising candidate for next generation wireless communications because of its ability of achieving high data rates at the cost of limited frequency bandwidth. When multiple users exist in the system, the capacity of MIMO system can be further improved due to multi-user gain.

We consider downlink case of a cellular system, which is also referred as MIMO broadcast channel (MIMO BC). Due to the non-degraded feature of multi-antennas broadcast channels, Dirty paper coding (DPC) is, to the best of our knowledge, the only way to achieve the capacity region of MIMO BCs. It is not convenient to study the whole capacity region of a broadcast system when multiple users exist, as the region is a high dimensional convex region. Many researchers turn to practical operating points of the capacity region. Most of published papers deal with sum rate capacity of MIMO BC [3].

The sum rate capacity is only a practical factor in packet

Manuscript received July 19, 2001; revised November 12, 2011. This work was supported in part by the S.K. Telecom.

Yingbo Li, Wei Jiang, Jianjun Zhang, Zhongjian Liu and Ye Jin are with the Satellite and Wireless Communication Laboratory, school of Electronics Engineering \& Computer Science, Peking University, Beijing 100871, P. R. China. E-mail: \{yingbo.li, jiangwei, zhangjj, quqianquhou and jinye\}@pku.edu.cn respectively. data scenarios, where it is possible to assign different rates among users. However, in circuit data scenarios, services demanded by users are sensitive to delay. Each user requires getting service from the base station at fixed rate. In this case, a practical performance evaluation factor is the user capacity. The user capacity is defined in [4] as the expected value of the number of users/receivers to which the transmitter can simultaneously transmit at an equal rate. Only the single antenna scenario is considered in [4].

Although, DPC is capacity achieving in MIMO BC, finding optimal users' covariance matrices and encoding order for maximization of user capacity introduces high complexity. Some researchers find the user capacity of MIMO BC by an exhaustive search of different groups of users and testing whether the desired rate vector is achievable according to their channel gain matrices and total power constraint. The complexity grows exponentially along with increasing of the number of users in the system.

Other suboptimal schemes can be found in [5], where fixed order of users is used. Users can be simply sorted by their indices, which are a representation of their arrival to the system and referred as first-come first serve (FCFS) scheme. In another scheme, users are sorted by ascending order of the power desired to achieve the target rate, which is referred as minimum power first (MPF) scheme. MPF scheme is shown in [5] to perform better than FCFS scheme with acceptable increase of complexity.

In this paper, we propose a better scheme based on DPC. The encoding order is no longer pre-determined like FCFS or MPF schemes, but iteratively settled. Different users are chosen successively in a greedy manner from a candidate set of users. The candidate user set concept is similar to best group concept for sum rate capacity calculation in [5], with the difference that the cardinality of candidate user set is fixed and the users in the set are dynamically adjusted. The search complexity can be controlled by adjusting cardinality of the candidate set.

The paper is organized as follows. System model is described in Section II. In Section III, general overview of user capacity evaluation is provided. In Section IV iterative user selection scheme is proposed. In Section V simulation results are given and in Section VI a conclusion.

\section{System Model}

Consider a MIMO BC with a base station (BS) employed with $t$ antennas and $K$ mobile users (MUs) with $r_{1} \ldots r_{K}$ antennas, respectively. The BS is required to send independent data streams to $K$ MUs constraint to a total 
sum power limitation of $P$. The channel model of MIMO Gaussian BC at any time sample is represented as follows for each user:

$$
\mathbf{y}_{i}^{r_{i} \times 1}=\mathbf{H}_{i}^{r_{i} \times t} \mathbf{x}^{t \times 1}+\mathbf{n}_{i}^{t \times 1}, i=1,2, \ldots, K
$$

In (1), $\mathbf{x}=\sum_{i=1}^{K} \mathbf{x}_{i}$, each $x_{i} \in C^{t \times 1}$ is signals intended for user $i$ respectively. $\mathbf{x}_{i}$ is assumed to have independent circular symmetric stationary complex Gaussian distribution, with zero mean and covariance matrix $\Sigma_{i}=\mathrm{E}\left[\mathbf{x}_{i} \mathbf{x}_{i}^{T}\right]$. The

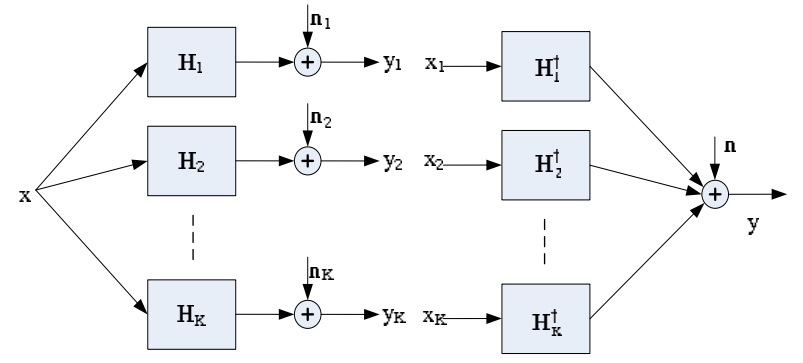

Fig. 1. Channel model of MIMO BC (left) and MIMO MAC (right)

input signal is subject to an total power constraint $P$, $\operatorname{tr}\left(\Sigma_{x}\right) \leq P$, where $\Sigma_{x}=\mathrm{E}\left[\mathbf{x} \mathbf{x}^{T}\right]=\Sigma_{1}+\Sigma_{2}+\ldots+\Sigma_{K}$ is the covariance matrix of the input signal, assuming independent signals intended for different users.

$\mathbf{H}_{i} \in C^{r_{i} \times t}$ is the complex channel gain matrix of user $i$. These matrixes are considered to be perfectly known at both the transmitter and the receiver. We consider $\mathbf{H}_{i}$ to be fixed during every channel use, i.e. we have enough time to estimate channel side information. The length of each frame may be very long to achieve good performance of Dirty Paper Coding. Entries of $\mathbf{H}_{i}$ have independent and identical complex Gaussian distribution with zero mean and unit variance $\sigma_{h}^{2}=1$, i.e. $C N(0,1)$.

$\mathbf{n}_{i} \in C^{r_{i} \times 1}$ is the complex additive white Gaussian noise at receiver side of user $i$. Entries of $\mathbf{n}_{i}$ are also independently and identically distributed with zero mean and unit variance.

The signal-to-noise ratio is defined as SNR $=S N R=P \sigma_{h}^{2} / \sigma_{n}^{2}=P$. Power limitation $P$ is adjusted for different SNR consideration in the following sections.

\section{USER CAPACITY EVALUATION}

In a circuit data transmitting system, service provided by the base station such as voice communication and video conference, is very sensitive to time delay. Each user demands to get service at a fixed rate. Without loss of generality, we assume all the users in the system desire the same fixed rate, denoted as $R_{0}$. The user capacity of a system is defined as the number of users it can serve simultaneously at rate $R_{0}$ desired by each user.

\section{A. Optimal Scheme}

The order of encoding matters a great deal in DPC. Under a particular encoding order and power constraint $\Sigma_{i}$ for every user, one can achieve a rate vector $\mathbf{R}\left(\pi, \Sigma_{i}\right)$ with entries

$$
R_{\pi(i)}=\log \frac{\left|\mathbf{I}+\mathbf{H}_{\pi(i)}\left(\sum_{j \geq i} \Sigma_{\pi(j)}\right) \mathbf{H}_{\pi(i)}^{T}\right|}{\left|\mathbf{I}+\mathbf{H}_{\pi(i)}\left(\sum_{j>i} \Sigma_{\pi(j)}\right) \mathbf{H}_{\pi(i)}^{T}\right|}, i=1, \cdots, K .
$$

where $\pi(i)$ is defined as a permutation function. The BC capacity region is

$$
C_{B C}(P, \mathbf{H}) \equiv C o\left(\bigcup_{\pi, \Sigma_{i}} \mathbf{R}\left(\pi, \Sigma_{i}\right)\right)
$$

The convex hull of the union is taken over all positive semi-definite covariance matrices $\Sigma_{i}$ and all permutations $\pi$, under power constraint $\operatorname{tr}\left(\Sigma_{x}\right)=\operatorname{tr}\left(\Sigma_{1}+\cdots+\Sigma_{K}\right) \leq P$.

This region is not a convex region, and it can be very difficult to solve this optimization problem. However, we can analyze it through a convex optimization problem due to the duality between $\mathrm{BC}$ and MAC.

This duality is proved in [7]. The channel matrix from every user to BS is $\mathbf{H}_{i}^{T}$ as in right part of Fig.1, and there is a total power constraint $\sum_{i=0}^{K} P_{i} \leq P$. The capacity of the dual MAC is

$$
\begin{aligned}
& C_{M A C}\left(P, \mathbf{H}^{T}\right) \equiv \bigcup C_{M A C}\left(P_{1}, \cdots, P_{K} ; \mathbf{H}^{T}\right) \\
& \sum_{i=0}^{K} P_{i} \leq P
\end{aligned}
$$

where

$$
\begin{aligned}
& C_{M A C}\left(P_{1}, \cdots, P_{K} ; \mathbf{H}^{T}\right) \equiv \bigcup_{t r\left(\mathbf{P}_{i}\right) \leq P_{i} \forall i}\left\{\left(R_{1}, \cdots, R_{K}\right):\right. \\
& \left.\sum_{i \in S} R_{i} \leq \log \left|\mathbf{I}+\sum_{i \in S} \mathbf{H}_{i}^{T} \mathbf{P}_{i} \mathbf{H}_{i}\right| \forall S \subseteq\{1, \cdots, K\}\right\} \\
& \text { In [7], the duality is established, } \\
& C_{M A C}\left(P, \mathbf{H}^{T}\right) \equiv C_{B C}(P, \mathbf{H})
\end{aligned}
$$

Thus, each rate achievable in MAC is also an achievable rate in $\mathrm{BC}$.

For a MIMO $\mathrm{BC}$, the rate vectors $\mathbf{R}=\left(r_{1}, \cdots, r_{K}\right)$ achieved using dirty paper coding by each users in the system form a convex set, $C_{B C}(P, \mathbf{H})$. The user capacity of MIMO $\mathrm{BC}$ can be calculated using Lemma 3 of [5], with which one can determine whether the rate vector $\left\{r_{1}, \cdots, r_{K}\right\}$ is achievable. Due to the convex character of the rate set, this is shown [5] to be a convex optimization problem, and can be solved by any standard algorithm. One can find the largest number of users by an exhaustive searching procedure. The corresponding DPC encoding order and covariance is decided as a by-product.

Although this strategy is optimal, it is of high complexity to find the optimal largest group of users. For example, in a system with $K$ users, to decide whether $\mathrm{m}$ is the user capacity, one has to exclude all rate vectors with $\mathrm{m}+1$ 
component of $R_{0}$ and to find some rate vector with $\mathrm{m}$ components of $R_{0}$ in the capacity region. The complexity grows exponentially with number of users $\mathrm{K}$ in the system.

Researchers in [5] impose fixed strategies on choosing these users to find a suboptimal user capacity. In [5], a predetermined encoding order is used, for example minimum power first (MPF) order.

\section{B. MPF Scheme}

In MPF scheme of [5], the users are encoded in increasing order of the power needed by them to achieve the desire rate $R_{0}$ seeing no interference from other users, i.e. single user case. The covariance matrices of each user are determined sequentially.

First sort the users in ascending power cost to achieve $R_{0}$, and choose the top $\mathrm{N}$ users. Assume users are already sorted as $1, \cdots, K$. User 1 consumes least power, and is encoded last to make sure it sees no interference from other users, $\mathbf{y}_{1}=\mathbf{H}_{1} \mathbf{x}_{1}+\mathbf{n}_{1}$. The desired $R_{0}$ is achieved by the base station using as less power as possible. The covariance matrix $\Sigma_{1}$ of $\mathbf{x}_{1}$ is found by an optimization problem by minimizing $\operatorname{tr}\left(\Sigma_{1}\right)$. This can be calculated either by a convex optimization algorithm or by standard water-filling algorithm. For user i, it is subject to interference from users that consume less power, whose covariance matrices already known as $\Sigma_{j, j}<i$.

$$
\mathbf{y}_{i}=\mathbf{H}_{i} \mathbf{x}_{i}+\mathbf{H}_{i} \sum_{j<i} \mathbf{x}_{j}+\mathbf{n}_{1}
$$

For calculating covariance matrix of user i, one can use the effective channel concept as in [7]. The effective channel gain matrix is defined as follows

$$
\tilde{\mathbf{H}}_{i}=\left[\mathbf{I}+\mathbf{H}_{i}\left(\sum_{\mathrm{j}<\mathrm{i}} \Sigma_{\mathrm{j}}\right) \mathbf{H}_{i}^{T}\right]^{-1 / 2} \mathbf{H}_{i}
$$

In this encoding order, the suboptimal user capacity is found to be

$$
N_{D P C}=\max \left\{N: \sum_{i=1}^{N} \operatorname{tr}\left(\Sigma_{i}\right) \leq P\right\}
$$

\section{ITERATIVE USER SELECTION SCHEME}

\section{A. Motivation}

The MPF scheme in [5] does not make sure an optimal encoding order or optimal covariance matrices of different users. Instead, it chooses ascending order of power as the ordering of users. The covariance matrix of the $i$ th user is optimized so as to minimize the power needed for it to achieve desire rate $R_{0}$. The covariance matrix optimal for this user may hurt other users, and it is just a local optimal choice.

After some users are chosen and their covariance matrices are determined, the relative order of the rest users may be different, treating message intended for the chosen ones as
Gaussian noise. The user consumes least power to achieve desired rate in single user case may not necessarily consumes least power seeing these Gaussian noise. Thus it is no longer necessary to stick to pre-determined order.

\section{B. Method}

We propose a better strategy using DPC for user capacity in a greedy manner. The transmitter chooses the users one by one. The order of users is not pre-determined, but adjusted promptly.

We first calculate the power needed for every user to transmit at the desired rate, choose the user that need the least power and assign it the corresponding transmit power. This user, we number it as user 1. It is encoded last using DPC to make sure it sees no interference from other users. Then we consider the power needed for the rest of users to transmit at the desired rate at the existence of interference from user 1 and choose the user (We may number it as user 2.) that needs the least power. For choosing the $i$ th user, the transmitter first computes power needed to achieve desired rate $R_{0}$ by each candidate, according to their channel gain matrices and covariance matrices $\Sigma_{1}, \ldots, \Sigma_{i-1}$ already assigned to the chosen users. This procedure is keeping on until there is not enough power to support any new users to transmit at the desired rate. Then we finally get the user capacity. The iterative user selection procedure is described as in Algorithm 1.

\section{Candidate Set Concept}

The algorithm described above search all the un-chosen users to find the best choice of next user. If the total number of users is large in the system, the searching for the best user will introduce much complexity. Meanwhile, in the MPF scheme, the order of users is totally fixed. It is much simple but not that flexible as our iterative user selection scheme. A compromise can be made to further reduce the searching complexity of our proposed scheme by candidate set concept.

We limit our search for the best user in the candidate user set instead of the entire user left. The search space is much smaller than that in Algorithm 1. Denote the cardinality of candidate user set $S$ as $T$, i.e. $T=|S|$. The value of $T$ can be adjusted for flexible compromise between performance and complexity. We can increase $T$ when the search complexity is not the major concern, and decrease it when the performance is beyond system requirement. With $T$ to be small, the searching complexity holds similarly the same for choosing different users.

We choose candidate user set according to each user's power consumption to achieve desired rate $R_{0}$ seeing no interference from others. Those $T$ users consuming least power are chosen dynamically each time to form the candidate set.

The performance is shown to be good in the following section. The reason is that: power needed to achieve desired rate seeing no interference shows the potential of one user to achieve better performance. The potential still serves a good indicator of its performance seeing interferences from the chosen users. 
When $T=1$, only one user is considered each time, and our iterative user selection scheme reduces to MPF scheme. When $T=K$, an exhaustive search over the un-chosen users is performed, as that in Algorithm 1.

\section{Algorithm.1 Iterative User Selection}

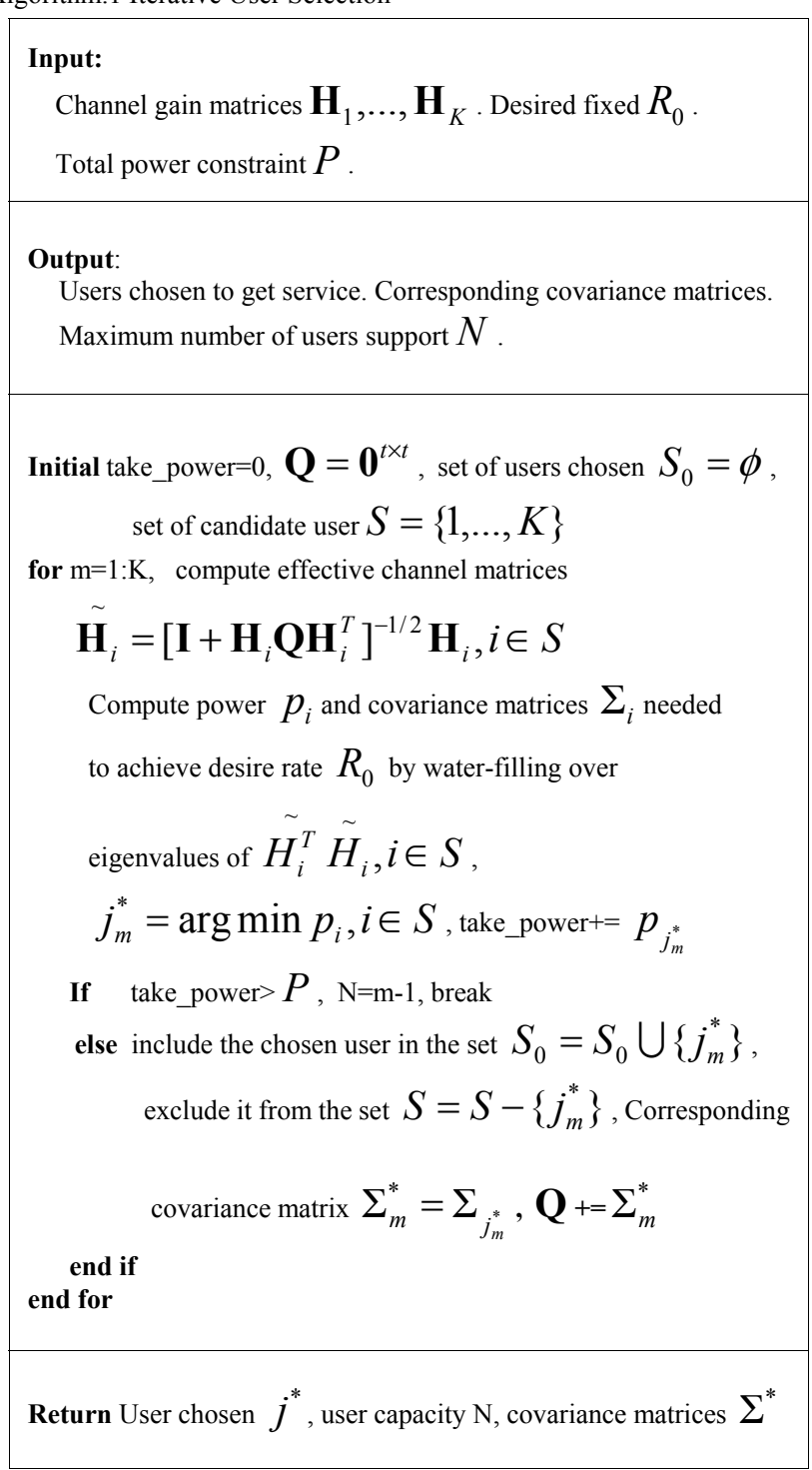

\section{P PERformance Simulation Results}

In this section we present simulation results of iterative user selection scheme and evaluation of its performance in comparison to the minimum power first scheme. We consider a MIMO broadcast channel with different number of users. Both the base station and the mobile users are equipped with four antennas. All the users in the system demand to be served by the base station at a fixed rate $R_{0}=3 \mathrm{bps} / \mathrm{Hz}$. Performances of our proposed scheme and MPF scheme are shown in Fig. 2. under two different channel SNR. When the number of users is large, our proposed scheme has a little advantage over MPF scheme.

From simulation we can see that our proposed scheme just have a little advantage over MPF scheme when both the BS and MU have equivalent number of antennas. However, in practical circumstance, the BS may be equipped with much more antennas than the MU. Performances of our proposed scheme are plotted in Fig. 3 and 4. The number of receiver antenna is fixed to be $r=1$, and $t=4$ and 8 respectively. We can see that implementing iterative user selection scheme brings significant improvement in user capacity. As the number of transmitter antennas grows, the advantage over MPF scheme becomes more significant.

Fig. 5 and 6 show the performance of our proposed scheme under different cardinality of candidate user set. A system with total of $\mathrm{K}=64$ users desiring fixed rate $R_{0}=3 \mathrm{bps} / \mathrm{Hz}$ is considered. The channel SNR is $38 \mathrm{~dB}$. From both Fig. 5 and Fig. 6, we can find that little enlargement of cardinality of $\mathrm{S}$ introduces great improvement of performance of our proposed scheme when $\mathrm{T}$ is small.

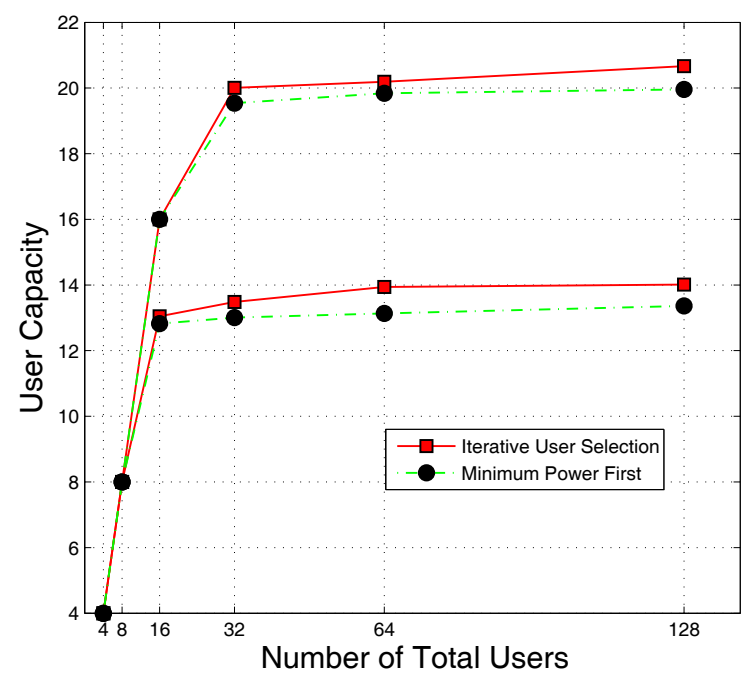

Fig. 2. User capacity at SNR $18,28 \mathrm{~dB}$, support $2 \mathrm{bps} / \mathrm{Hz}$ for each user, antenna configuration $\mathrm{t}=4, \mathrm{r}=4$. The top two lines are user capacity at $\mathrm{SNR}$ $28 \mathrm{~dB}$, and the rest two $18 \mathrm{~dB}$.

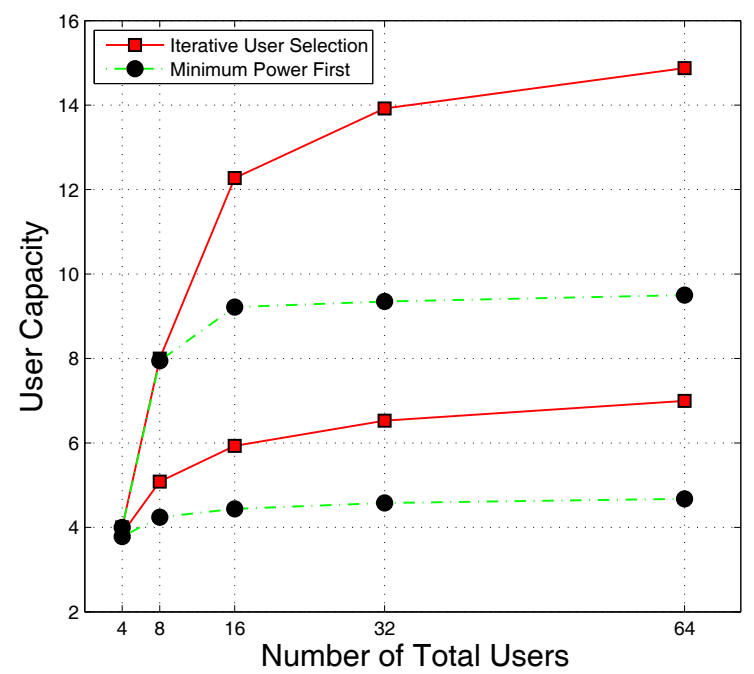

Fig. 3. User capacity at SNR $18,38 \mathrm{~dB}$, support $3 \mathrm{bps} / \mathrm{Hz}$ for each user, antenna configuration $\mathrm{t}=4, \mathrm{r}=1$. The top two lines are user capacity at SNR $38 \mathrm{~dB}$, and the rest two $18 \mathrm{~dB}$.

\section{CONCLUSION}

In this paper we study the problem of maximizing user capacity of MIMO Gaussian BCs in circuit scenarios. We 
have presented a new scheme based on iterative user selection. The order of users is determined iteratively in a greedy manner. Simulation results showed that the proposed scheme have significant advantage over MPF scheme when the base station is equipped with much more antennas than mobile stations. A compromise between performance and complexity can be made using a candidate user set concept.

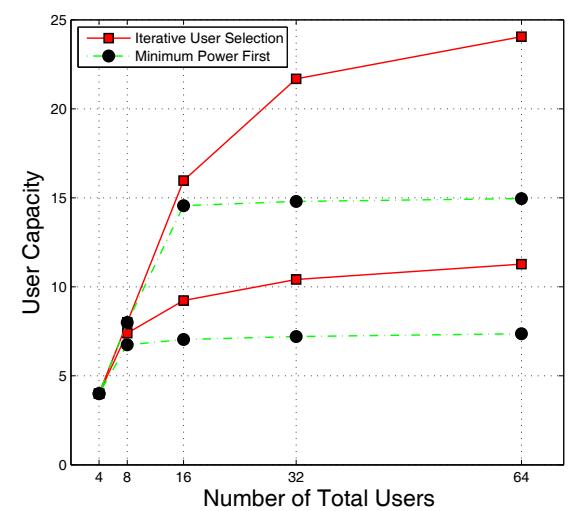

Fig. 4. User capacity at SNR $18,38 \mathrm{~dB}$, support $3 \mathrm{bps} / \mathrm{Hz}$ for each user, antenna configuration $\mathrm{t}=8, \mathrm{r}=1$. The top two lines are user capacity at SNR $38 \mathrm{~dB}$, and the rest two $18 \mathrm{~dB}$.

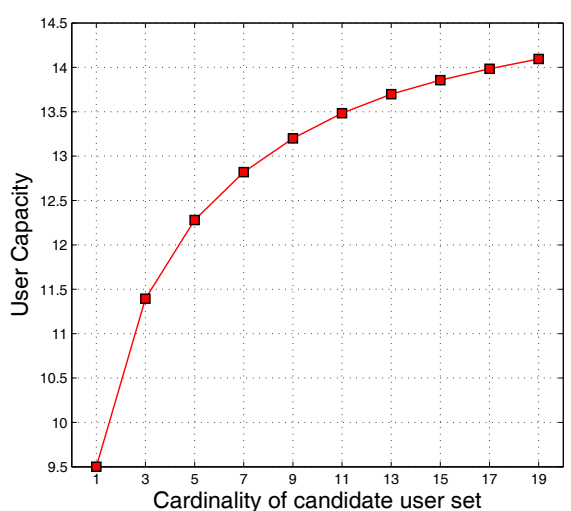

Fig. 5. User capacity at different cardinality of candidate user set, at SNR $38 \mathrm{~dB}$, total number of users 64 , support $3 \mathrm{bps} / \mathrm{Hz}$ for each user, antenna configuration $\mathrm{t}=4, \mathrm{r}=1$.

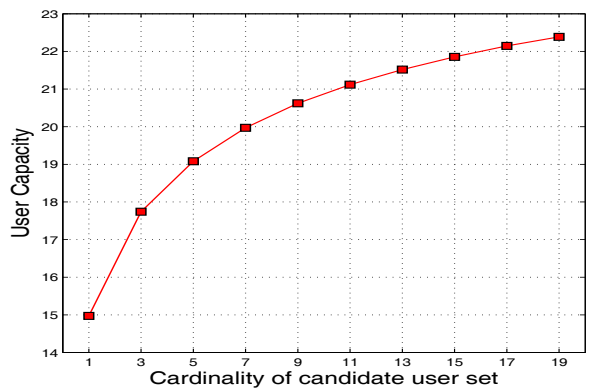

Fig. 6. User capacity at different cardinality of candidate user set, at SNR $38 \mathrm{~dB}$, total number of users 64 , support $3 \mathrm{bps} / \mathrm{Hz}$ for each user, antenna configuration $\mathrm{t}=8, \mathrm{r}=1$

\section{ACKNOWLEDGMENT}

Yingbo Li thanks the anonymous reviewers from ICFCC, whose comments help us a lot improve this paper.

\section{REFERENCES}

[1] I. Telatar, "Capacity of multi-antenna gaussian channels," Bell Laborato-ries, Tech. Rep., June 1995.

[2] G. J. Foschini and M. J. Gans, "On limits of wireless communications in a fading environment when using multiple antennas," Wireless Personal Communications, vol. 6, pp. 311-335, 1998.

[3] G. Caire and S. Shamai, "On the achievable throughput of a multiantenna gaussian broadcast channel," IEEE Transactions on Information Theory, vol. 49, no. 7, pp. 1691-1706, July 2003.

[4] S. Boppana and J. Shea, "Downlink user capacity of cellular systems: Tdma vs dirty paper coding," in Information Theory, 2006 IEEE Inter-national Symposium on, July 2006, pp. 754-758.

[5] H. Viswanathan, S. Venkatesan, and H. Huang, "Downlink capacityevaluation of cellular networks with known-interference cancellation," Selected Areas in Communications, IEEE Journal on, vol. 21, no. 5, pp. 802-811, June 2003.

[6] G. Aniba and S. Aissa, "Multi-user capacity maximization for mimogaussian broadcast channels," in Communications, 2006. ICC '06. IEEEInternational Conference on, vol. 9, June 2006, pp. 4196-4201.

[7] S. Vishwanath, N. Jindal, and A. Goldsmith, "Duality, achievable ratesand sum-rate capacity of gaussian mimo broadcast channels," IEEETransactions on Information Theory, vol. 49, pp. 2658-2668, October2003.

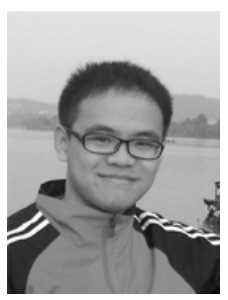

Yingbo $\mathbf{L i}$ received the B.S. degrees in information science and technology from Beijing Normal University, Beijing, China, in 2008.

$\mathrm{He}$ is now a Ph.D. candidate in electrical engineering in school of Electronics Engineering \& Computer Science, Peking University, Beijing, China. His research interests include wireless communications/networks, next generation communication technology and signal processing.
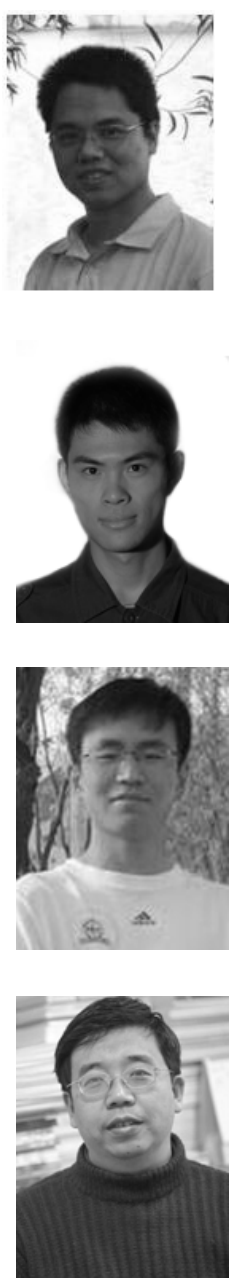

Wei Jiang received the B.S. and Ph.D. degrees in electrical engineering from school of Electronics Engineering \& Computer Science, Peking University, Beijing, China, in 1996 and 2001, respectively. $\mathrm{He}$ is now an associate professor in school of Electronics Engineering \& Computer Science, Peking University, Beijing, China. His research interests include wireless communications/networks, next generation communication technology, channel coding, modulation technique, and signal processing.

Jiangjun Zhang received the B.S. degrees in information science and technology from Nankai University, Tianjin, China, in 2009

$\mathrm{He}$ is now a Ph.D. candidate in electrical engineering in school of Electronics Engineering \& Computer Science, Peking University, Beijing, China. His research interests include channel coding, channel estimation and signal processing.

Zhongjian Liu received the B.S. degrees in information science and technology from Nankai University, Tianjin, China, in 2009.

$\mathrm{He}$ is now a Ph.D. candidate in electrical engineering in school of Electronics Engineering \& Computer Science, Peking University, Beijing, China. His research interests include MIMO communication, modulation technique, and advanced communication schemes.

Ye Jin received the B.S. and M.S. degrees in electrical engineering from school of Electronics Engineering \& Computer Science, Peking University, Beijing, China, in 1989 and 1992, respectively.

$\mathrm{He}$ is now a professor in school of Electronics Engineering \& Computer Science, Peking University, Beijing, China. His research interests include satellite communications/networks, next generation communication technology, signal processing. 